\title{
Study on the Relationship between Melody Perception and Music Harmonics
}

\author{
Fajiang $\mathrm{Ma}^{1}$, Geng $\operatorname{Tian}^{2}$, Lan Tian ${ }^{1, *}$, Xiaoshan $\mathrm{Lu}^{3}$ and Shuzhong Bai ${ }^{4}$ \\ ${ }^{1}$ School of Microelectronics, Shandong University, Jinan, Shandong, China \\ ${ }^{2}$ School of Life Science, Shandong University, Jinan, Shandong, China \\ ${ }^{3}$ School of information science and engineering, Shandong University, Jinan, Shandong, China \\ ${ }^{4}$ School of electrical engineering, Shandong University, Jinan, Shandong, China \\ *Corresponding author
}

\begin{abstract}
Melody is the key perception of music. For the heavy sensory nerve deafness, the current cochlear implant (CI) user has the clinical effect on speech recognition, but the music melody and timbre perception is not acceptable, they could not normally perceive music information. Based on CI encoding principle of multi-channel filtering and music harmonics analysis and synthesis technology, this paper studies the relationship between melody perception and harmonic structure of music signal. Utilizing Fourier transform, the original music signal spectral were analyzed, the selected harmonic components were filtered and then rebuilt the synthesized sounds by Fourier inversion transform. Several single music note and melody segments audiometry tests were experimented by normal hearings. The test results show that the fundamental frequency was the decisive component of pitch perception, lacking other few harmonics affected pitch perception much slightly, but large numbers of harmonic absence deteriorated music quality and consequently affected pitch perception, even the fundamental wave still remained, which might relate to the destruction of spectral structure. So it is concluded that music melody perception is related to the signal channels of multi-channel CI or electrodes distribution, good music perception should based on reasonable electrodes distribution. The study results suggested that the electrodes distribution should be optimized and electrode number should be increased properly in cochlea locations corresponding to the characteristic frequency of music basic notes.
\end{abstract}

Keywords-pitch perception; cochlear implant (CI); music melody; harmonics; electrode

\section{INTRODUCTION}

Music perception has a poor effect currently and still remains unsolved in cochlea implants(CI)[1]. Modern CI processors employing the method of extracting the envelop of the sound could realize the purpose of speech recognition. Music signals have a more widespread frequency range, and speech contains large amounts of redundant information. Speech recognition wouldn't be limited even lack of the redundant information, such like rhythm, pitch and timbre, which were extremely important for enjoying music.

Melody, harmony, rhythm, and timbre consist a set of elementary properties of music, which is widely accepted [2]. While without distinctive rhythm and lyrics, melody perception ability is much poor among CI users, which is largely dependent on the ability of pitch perception. The pitch sensing mechanism of harmonic complex tones in normal hearing is still in the research stage [3], which could provide positive references to CI pitch perception. The fine structure is most important for pitch perception [4], and some newly proposed processing strategy has taken the fine structure into account, such like the FSP of Med-EL company [5]. In theory, both the fundamental frequency and the bandwidth of adjacent harmonics could represent the pitch [2]. Timbre is described as "the color of music instrument", and various distinctive timbre would express wealthy emotion. Early theory thinks that spectral envelop dominates the timbre. The absence of some harmonics with high energy or certain harmonic probably causes the variation of spectral envelop, which changes the timbre, thus both the fundamental wave and the harmonics spacing would possibly impact the timbre perception. Recent theory thinks that the spectral shape couldn't completely determine the timbre and its perception also relates to temporal clues [6]. The example of Vowel /i/ and /a/ sung by same singer shows that different spectral envelops could represent the same timbre. Spectral attributes naturally correlate with the fine structure of acoustic signals [7]. Therefore, either spectral distribution or temporal waveform is changed, and the other will be changed accordingly.

From the aspect of frequency domain, to investigate the mechanism of complex tones perception, applying the reconstruction method based on signal spectral energy, through controlling the harmonics constitution of the synthesized tones, this paper mainly studied the influences of the fundamental wave and adjacent harmonics spacing on pitch and timbre perception. Several audiometry experiments with normal hearing subjects was set, and the correlation between temporal fine structure and spectral attributes was investigated.

\section{SignAl PROCESSING}

After dealing with spectrum analysis, the input signal was processed by Fourier transform, and harmonic amplitudes were substituted by the average value of FFT point amplitudes nearby the central frequency of corresponding harmonic, at last the synthesized signal was constituted by selected harmonics finally. The original signal can be expressed as equation (1).

$$
s(n)=\sum_{i=1}^{k} A_{i} \cos \left(n \omega_{i}+\varphi_{i}\right)
$$

where $A_{i}$ represents the harmonic amplitude, $\varphi_{i}$ is the harmonic phase, and $\omega_{i}$ represents its central frequency. For the correlation between spectral distribution and temporal fine 
structure, the frequency $\omega_{i}$ not only corresponds to the electrode stimulation position and also relates to stimulation rates dependent on place.

\section{AUDIOMETRY EXPERIMENT AND ANALYSIS}

All the experiment music notes were amplitude normalized to exclude the influence of sound volume, and chosen from the frequency range of $200-2 \mathrm{KHz}$. The sampling frequency was $16 \mathrm{KHz}$ and the sampling precision is 16bits. A simple test was set to examine whether subjects had favorable pitch discrimination ability before the audiometry test. At last, ten subjects participated the test, including 5 males and 5 females, and nine subjects took part in the whole experiment. All the tests were performed in quiet laboratory using closed headphone (Sennheiser HAD 200). During the test, subjects were required to rank the pitch of two tones and describe the difference on timbre.

\section{A. Impact of Odd and Even Harmonics on Music Note}

Single notes G3, C4, A4, D5, B5, and E6 of two kinds of instruments (piano and clarinet) was selected, to make sure that no frequency doubling phenomenon existed among the pitches of test notes. The spectral envelop of piano note has a relatively flat structure and average harmonic amplitudes. However, clarinet has a distinctive spectral envelop, that the energy of odd harmonics is strong and the even ones is much weaker. Table 1 shows the results of pitch comparison test. Results shows that the pitch of full-harmonics tone barely changes, which verifies the effectiveness of the reconstruction method. While neglecting the influence of timbre difference, the pitches of full-evenharmonics tones was considered higher than the original notes. Majority think that the pitch of full-odd-harmonics tones have not changed.

Figure 1 shows the spectrogram, from which we find that the spectral structure of full-harmonics tone barely changed, the adjacent harmonics spacing of full-odd-harmonics and fulleven-harmonics tone has been doubled, and the first wave of full-even-harmonics is up-shifted. The results of pitch comparison explain the impact of fundamental wave location on pitch perception, that up-shift of the first wave increased pitch. Removing odd or even harmonics to enlarge the harmonics spacing destroyed the spectrum integrity, so that the tone quality would be changed which might disturb the pitch comparison tests. The impact of adjacent harmonics spacing couldn't be confirmed. The fundamental wave location on the frequency band is represented by the electrode stimulation place on cochlea, thus effective electrodes design method should conform the distribution of note frequency, in order to perceive relatively accurate pitch among CI users.

TABLE I. RESULT OF PITCH COMPARISON

\begin{tabular}{|l|c|c|c|}
\hline \multirow{2}{*}{$\begin{array}{c}\text { Pitch } \\
\text { comparison to } \\
\text { original tone }\end{array}$} & Full Harmonics & $\begin{array}{c}\text { Full-Odd- } \\
\text { Harmonics }\end{array}$ & $\begin{array}{c}\text { Full-Even- } \\
\text { Harmonics }\end{array}$ \\
\cline { 2 - 4 } Higher & 0 & $15 \%$ & $85 \%$ \\
\hline Lower & $10 \%$ & $20 \%$ & $5 \%$ \\
\hline Same & $90 \%$ & $65 \%$ & $10 \%$ \\
\hline
\end{tabular}

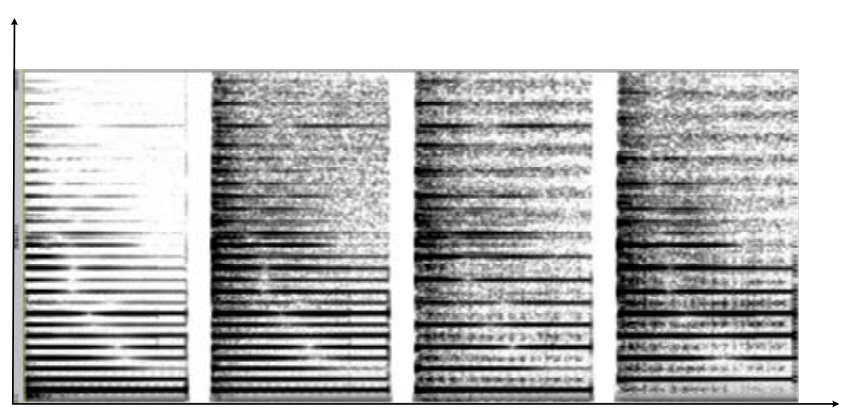

FIGURE I. SPECTROGARAM OF THE ORIGINAL,FUUL-HARMONICS, FULL-ODD-HARMONICS AND FULL-EVEN-HARMONICS TONES

In the timbre recognition test of synthesized tones, which is shown in Figure 2, full-harmonics tone could be recognized well, while the recognition results of full-odd-harmonics and fulleven-harmonics tones are unsatisfactory. Large amounts of harmonics removed affects the spectral integrity and then causes timbre changing. The possible reason why CI users have bad timbre perception effect is that numbers of harmonics can't be transmitted or harmonics are transmitted to malposition in cochlea. Due to the special spectral structure of clarinet, the timbre recognition is poor enough. Besides, the high-pitched tones have less harmonics, so that timbre is much harder to recognize.

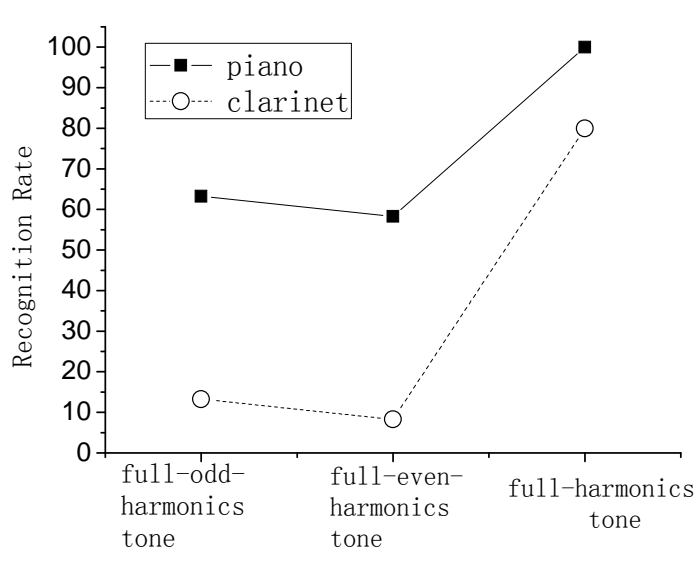

\section{B. Impact of Crucial Single Harmonics}

To specifically verify the impact on pitch perception of crucial single harmonic, the audiometry test of synthesized tones lacking single harmonic only was set. Melody recognition test was performed to research the influence of the fundamental wave on melody perception. The major harmonics in the low frequency band have relatively higher energy, the impact of which is deserved to be investigated, the fundamental wave especially. Figure 3 shows the audiometry results of the impact of the fundamental harmonic, the second, third, fourth, and fifth harmonics on note pitch perception. Compared with the original tone, about $79.6 \%$ synthesized notes lacking fundamental wave only have higher pitches, and the pitch difference is getting more obvious while the pitch of its corresponding original tone is higher. Subjects described that there existed a significantly 
timbre difference between the original tones and the synthesized ones that lacking fundamental wave, and the latter is more similar to a metallic sound. While lacking other harmonics only, pitch and timbre of the synthesized tones almost remained unchanged.

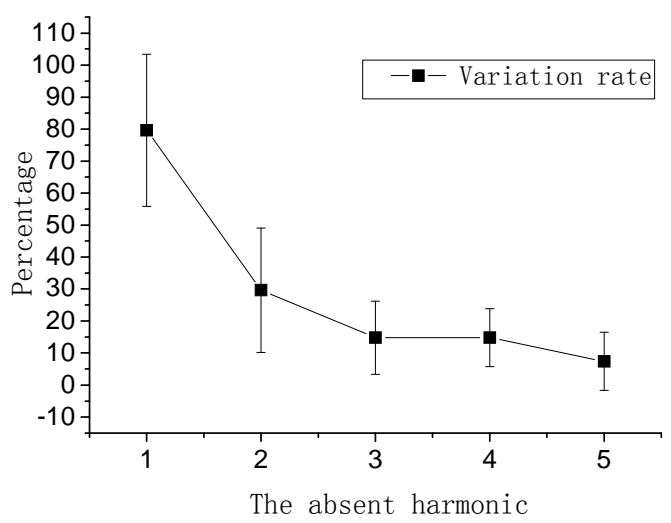

FIGURE III. RATE OF PITCHCHANGES.

Seven simple melody consisting of three equal-time notes without obvious rhythm was recorded using professional software (Gold Wave v5.67). The means of 3 note frequencies of each melody were arranged in ascending order at intervals of four semitones. A random note of each test melody was processed with removing the fundamental wave. Melodies were tested in a random order among subjects, and the results was shown in Figure 4. While the average pitch of the melody was relatively low, the melody could be recognized well along with the lack of one note's fundamental wave (the recognition rate was $89 \%$ ). However, along with the rising of the average pitch, the recognition rate declined remarkably, and the average recognition rate was only $42.9 \%$. Above experiments showed that, fundamental wave significantly affected pitch and timbre perception of music notes, and further influence melody perception.

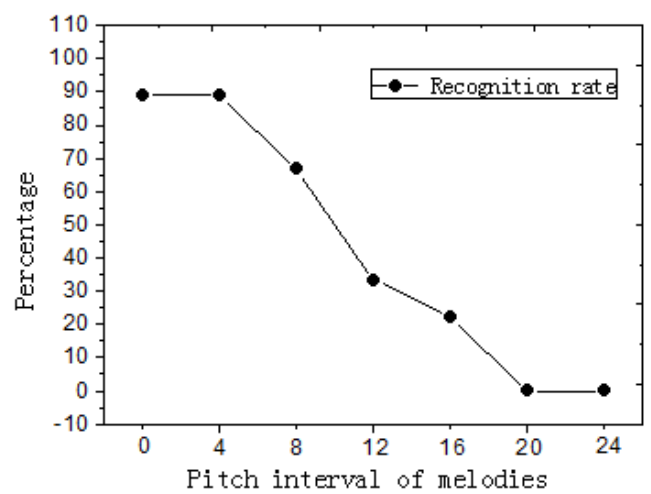

FIGURE IV. EXAMPLE OF A FIGURE CAPTIONRATES OF MELODY RECOGNITION .

\section{CORRELATION BETWEEN SPECTRUM AND TEMPORAL FINE STRUCTURE}

The timbre difference among synthesized tones disturbed the subjective pitch ranking tests partly. Analysis to temporal waveform was necessarily to be performed, and the correlation between spectral attributes and temporal fine structure could also be illustrated to some extent. After the fine waveforms of fullharmonics and full-odd-harmonics and full-even-harmonics tones of several notes were observed and measured, the results found that the full-harmonics and full-odd-harmonics tone had the same pitch with the original tone, but the pitch of full-evenharmonics tone is twice in the range of the errors permitted. Therefore, doubling the spectral spacing didn't alter the pitch of full-odd-harmonics tone, and "up-shift" of fundamental wave doubled the pitch of the full-even-harmonics tone. Removing fundamental wave altered the structure of spectral distribution and temporal fine structure simultaneously, which was resulted from their inherent correlation.

\section{CONCLUSION}

Above results show that the fundamental wave is the critical component for pitch or melody perception from the aspect of frequency domain. The adjacent harmonics spacing barely affects pitch perception, yet timbre can't be perceived well when lacking amounts of harmonics. In order to perceive music better, the fundamental wave should be conveyed in priority and other harmonics should be delivered as much as possible. The design scheme of electrodes array should conform the pitched distribution of music notes and should be closed to the frequency regions both the fundamental frequency as precise as possible and the other harmonic frequencies as much as possible.

\section{ACKNOWLEDGMENT}

This research was sponsored by the National Science Foundation of China (No.11474185) and Shandong province key research plan (No. 2016GGX101028).

\section{REFERENCES}

[1] C. Olszewski, E. Gfeller, R. Forman and et al, "Familiar melody recognition by children and adults using cochlea implants and normal hearing children,” Cochlea Implants International, 6th ed, vol. 3, 2004, pp. 123-140.

[2] Fan-Gang Zeng, Arthur N. popper, and Richard R. Fay, “Auditory Prostheses - New Horizons”, Springer, 2011.

[3] Wang Jian, Guan Tian, and Ye Datian, "Pitch perception of harmonic complex tones based on excitation pattern,” ACTA ACUSTICA, 38th ed, vol 1, 2013, pp:99-104.

[4] Zachary M S, Bertrand D, and Andrew J O, "Chimaeric sounds reveal dichotomies in auditory perception,” Nature, 416th ed, vol 6876, 2002, pp:87-90.

[5] Hochmair I, Nopp P, Jolly C, and et al, "MED-EL cochlear implants: state of the art and a glimpse into the future”, Trends Amplif, 10th ed, vol. 4, 2006, pp201-219.

[6] McDermott HJ, "Music perception with cochlear implants: a review”, Trends Amplif, 8th ed, vol. 2, 2004, pp:49-82.

[7] Tobias Rader, Julia Doge, Uwe Baumann, and et al, "place dependent stimulation rates improve pitch perception in cochlea implantees with single-sided deafness”, Hearing Research, 339th ed, 2016, pp:94-103. 